\title{
Heat Kernel Estimates and Parabolic Harnack Inequalities on Graphs and Resistance Forms ${ }^{\dagger}$
}

\author{
By
}

\author{
Takashi KUmAGA *
}

\begin{abstract}
We summarize recent work on heat kernel estimates and parabolic Harnack inequalities for graphs, where the time scale is the $\beta$-th power of the space scale for some $\beta \geq 2$. We then discuss self-adjoint operators induced by resistance forms. Using a resistance metric, we give a simple condition for detailed heat kernel estimates and parabolic Harnack inequalities. As an application, we show that on trees a detailed two-sided heat kernel estimate is equivalent to some volume growth condition.
\end{abstract}

\section{$\S 1$ Introduction: History in Brief}

There is a long history of the study of detailed heat kernel estimates. For any divergence operator $\mathcal{L}=\sum_{i, j=1}^{n} \frac{\partial}{\partial x_{i}}\left(a_{i j}(x) \frac{\partial}{\partial x_{j}}\right)$ on $\mathbb{R}^{n}$ satisfying a uniform elliptic condition, Aronson [1] proved that the heat kernel $p_{t}(x, y)$ satisfies the two-sided Gaussian bound

$$
c_{1} t^{-n / 2} \exp \left(-\frac{d(x, y)^{2}}{c_{1} t}\right) \leq p_{t}(x, y) \leq c_{2} t^{-n / 2} \exp \left(-\frac{d(x, y)^{2}}{c_{2} t}\right)
$$

where $d$ is the Euclidean metric. Later in the 20th century, there were various outstanding results in the field of global analysis on manifolds. Let $\Delta$ be the Laplace-Beltrami operator on a complete Riemannian manifold $M$ with the Riemannian metric $d$ and with the Riemannian measure $\mu$. Li-Yau [41] proved a remarkable fact that if $M$ has non-negative Ricci curvature, then the heat

Communicated by Y. Takahashi, Received November 10, 2003. Revised December 22, 2003.

2000 Mathematics Subject Classification(s): Primary 60J35; Secondary 31B05, 60J10

This research is supported in part by the Inamori Foundation.

${ }^{\dagger}$ This article is an invited contribution to a special issue of Publications of RIMS commemorating the fortieth anniversary of the founding of the Research Institute for Mathematical Sciences.

*RIMS, Kyoto University, Kyoto 606-8502, Japan. Current address: Department of Mathematics, Malott Hall, Cornell University, Ithaca, NY 14853, USA

(C) 2004 Research Institute for Mathematical Sciences, Kyoto University. All rights reserved. 
kernel $p_{t}(x, y)$ satisfies

$$
\begin{gathered}
\frac{c_{3}}{\mu\left(B\left(x, t^{1 / 2}\right)\right)} \exp \left(-\frac{d(x, y)^{2}}{c_{3} t}\right) \leq p_{t}(x, y) \\
\leq \frac{c_{4}}{\mu\left(B\left(x, t^{1 / 2}\right)\right)} \exp \left(-\frac{d(x, y)^{2}}{c_{4} t}\right) .
\end{gathered}
$$

A few years later, Grigor'yan [23] and Saloff-Coste [46] elegantly refined the result and proved, in conjunction with the results by Fabes-Stroock [20] and Kusuoka-Stroock [39], that (1.2) is equivalent to a volume doubling condition (VD) plus Poincaré inequalities $(\mathrm{PI}(2))$ - see $\S 2.2$ for definitions in the graph setting. The results were then extended to the framework of Dirichlet forms by Sturm [50, 51] and Biroli-Mosco [14], to the framework of graphs by Delmotte [19].

In fact, detailed heat kernel estimates are strongly related to the control of harmonic functions, i.e. elliptic and parabolic Harnack inequalities (EHI), (PHI(2)) on M. The origin of ideas and techniques used in this field go back to Nash [45], Moser [43, 44] and there are many other significant works in this area (see [13, 18, 47] etc. for details). Summarizing, the following equivalence holds.

$$
(1.2) \Leftrightarrow(V D)+(P I(2)) \Leftrightarrow(P H I(2)) .
$$

An important corollary of this fact is, as (VD) and (PI(2)) are stable under certain perturbations of the operator, (1.2) and (PHI(2)) are also stable under these perturbations.

Since the late 1980's, there has been a lot of development in the mathematical study of diffusion processes and corresponding Laplace operators on fractals. The motivation behind this area comes originally from the work of mathematical physicists on heat decays for disordered media such as polymers and networks [29]. They found some kind of self-similar structures in the models and made various computations on "ideal" disordered media such as Sierpinski gaskets, that have exact self-similarity and nice symmetry.

Through the mathematical study of analysis on fractals, it turns out that for some class of "nice" fractals (for example the Sierpinski gasket), the Laplace operator is suitably defined and the heat kernel satisfies the following subGaussian bound,

$$
\begin{gathered}
c_{5} t^{-\alpha / \beta} \exp \left(-\left(\frac{d(x, y)^{\beta}}{c_{5} t}\right)^{1 /(\beta-1)}\right) \leq p_{t}(x, y) \\
\leq c_{6} t^{-\alpha / \beta} \exp \left(-\left(\frac{d(x, y)^{\beta}}{c_{6} t}\right)^{1 /(\beta-1)}\right),
\end{gathered}
$$


where $\alpha$ is a Hausdorff dimension and $\beta \geq 2$ is called a walk dimension on the fractal. For the case of the Sierpinski gasket, $\alpha=\log 3 / \log 2$ and $\beta=\log 5 / \log 2$. In a sense, the Laplace operator of the fractal is a " $\beta$-th order" operator instead of second order. Rigorously speaking, the domain of the corresponding Dirichlet form (quadratic form) is a Besov space of order $\beta / 2$, instead of the Sobolev space of order 1. Thus, some of the important classical methods of analysing heat kernels (such as the Davies method, the Moser's iteration) do not work and new methods are needed to analyse them. In addition, there are various properties of diffusion processes/Laplace operators on fractals which differ completely from those on the Euclidean spaces (see [2, 4, 35, 38, 49] etc. for details).

Thus, it is natural and important to search for conditions equivalent to (1.4), especially conditions which are stable under perturbations. This direction of research was developed quite recently. At this stage, most of the results are on graphs and general measure metric space versions are in preparations. In this paper, we will give a survey of the work in section 2 and we will give some new equivalence conditions under the framework of resistance forms in section 3. Especially, on trees we show that detailed two-sided heat kernel estimate is equivalent to some volume growth condition (Proposition 5.1).

We believe that the detailed study of sub-Gaussian heat kernel estimates and parabolic Harnack inequalities is relevant to global analysis on manifolds and measure metric spaces. Further, it is also relevant to the study of heat transfer on stochastic models such as percolation clusters.

Throughout the paper, $f \simeq g$ means that there exists $c_{1}, c_{2}>0$ such that $c_{1} f(x) \leq g(x) \leq c_{2} g(x)$ for all $x$.

\section{$\S 2 . \quad H e a t$ Kernel Estimates and Harnack Inequalities on Graphs}

In this section, we summarize recent work on heat kernel estimates and parabolic Harnack inequalities on graphs.

\section{§2.1. Framework}

Let $\Gamma$ be a infinite connected locally finite graph. Assume that the graph $\Gamma$ is endowed with a weight (conductance) $\mu_{x y}$, which is a symmetric nonnegative function on $\Gamma \times \Gamma$ such that $\mu_{x y}>0$ if and only if $x$ and $y$ are connected by a bond (in which case we write $x \sim y$ ). We call the pair $(\Gamma, \mu)$ a weighted graph. We can regard it as an electrical network. 
We define a quadratic form on $(\Gamma, \mu)$ as follows. For each $f, g \in \mathbb{R}^{\Gamma}:=\{h$ : $h$ is a function on $\Gamma\}$, we set

$$
\mathcal{E}(f, g)=\frac{1}{2} \sum_{\substack{x, y \in \Gamma \\ x \sim y}}(f(x)-f(y))(g(x)-g(y)) \mu_{x y} .
$$

We say $\left(\Gamma, \mu^{\prime}\right)$ is a bounded perturbation of $(\Gamma, \mu)$ if $\mu_{x y} \simeq \mu_{x y}^{\prime}$ for all $x \sim y$.

Now, define $\mu_{x}=\sum_{y \in \Gamma} \mu_{x y}$ for each $x \in \Gamma$. Set $\mu(A)=\sum_{x \in A} \mu_{x}$ for each $A \subset \Gamma ; \mu$ is then a measure on $\Gamma$. For each $x \sim y$, define $P(x, y)=$ $\mu_{x y} / \mu_{x}$, which is then the transition probability matrix of the Markov chain corresponding to $\mathcal{E}$. To be precise, the Markov chain corresponding to $\mathcal{E}$ is a continuous time Markov chain in which jumps occur along edge $\{x, y\}$ at rate $\mu_{x y}$. In this section, we will consider instead the induced random walk, the discrete time Markov chain which moves at unit time intervals to any vertex $y$ in the neighbourhood of $x$ with probabilities given by $\{P(x, y)\}$, since its long time asymptotic behaviour is similar to that of the continuous time Markov chain. We denote the induced random walk by $\left\{X_{n}\right\}_{n \geq 0}$. The random walk is reversible with respect to $\mu$, indeed,

$$
P(x, y) \mu_{x}=\mu_{x y}=\mu_{y x}=P(y, x) \mu_{y} .
$$

The discrete Laplace operator corresponding to the random walk is

$$
\mathcal{L} f(x)=\sum_{y} P(x, y) f(y)-f(x)=\frac{1}{\mu_{x}} \sum_{y}(f(y)-f(x)) \mu_{x y} .
$$

Let $P_{n}(x, y)$ be the transition function of the random walk $X_{n}$, i.e.

$$
P_{n}(x, y)=\mathbb{P}^{x}\left(X_{n}=y\right) .
$$

Define the heat kernel of $\mathcal{L}$ (transition density of $X_{n}$ ) by $p_{n}(x, y):=P_{n}(x, y) / \mu_{y}$. Clearly, $p_{n}(x, y)=p_{n}(y, x)$.

The natural metric on the graph obtained by counting the number of steps in the shortest path between points is written $d(x, y)$ for $x, y \in \Gamma$.

\section{§2.2. Recent results on heat kernel estimates and parabolic Harnack inequalities on graphs}

In this subsection, we will explain the results in $[8,12,24,25,28]$. Throughout this and the next subsections, we use the graph distance $d(\cdot, \cdot)$ and denote $B(x, r)=\{y \in \Gamma: d(x, y)<r\}, V(x, r)=\mu(B(x, r))$. We note that there are also related recent work for heat kernel estimates on graphs with sub-Gaussian heat transfer (cf. [3, 9, 31, 52, 53] etc.). 
Definition 2.1. Let $(\Gamma, \mu)$ be a weighted graph and let $\beta>0$.

(1) We say $(\Gamma, \mu)$ satisfies a $\left(p_{0}\right)$ condition if there exists $p_{0}>0$ such that

$$
p_{x y}=\mu_{x y} / \mu_{x} \geq p_{0} \quad \text { for all }\{x, y\} \in B .
$$

(2) We say $(\Gamma, \mu)$ satisfies a volume doubling condition $(V D)$ if there exists $C_{1}>1$ such that

$$
V(x, 2 R) \leq C_{1} V(x, R) \quad \text { for all } x \in \Gamma, R \geq 1 .
$$

(3) We say $(\Gamma, \mu)$ has walk dimension $\beta$ and $(\Gamma, \mu)$ satisfies $\left(E_{\beta}\right)$ if

$$
E^{x}\left[T_{B(x, R)}\right] \simeq R^{\beta}, \quad \text { for all } R \in[1, \infty), x \in \Gamma,
$$

where $T_{B(x, R)}:=\inf \left\{n \geq 0: X_{n} \notin B(x, R)\right\}$.

(4) We say $(\Gamma, \mu)$ satisfies an elliptic Harnack inequality $(E H I)$ if there exists $C_{1}>0$ such that, whenever $x \in \Gamma, R \geq 1$ and $h: \Gamma \rightarrow \mathbb{R}$ is non-negative and harmonic in $B(x, 2 R)$,

$$
\sup _{y \in B(x, R)} h(y) \leq C_{1} \inf _{y \in B(x, R)} h(y) .
$$

(5) We say $(\Gamma, \mu)$ has resistance exponent $\beta$ and $(\Gamma, \mu)$ satisfies $\left(R_{\beta}\right)$ if

$$
R\left(B(x, R), B(x, 2 R)^{c}\right) \simeq \frac{R^{\beta}}{V(x, R)}, \quad \text { for all } R \geq 1
$$

where resistance between disjoint sets $A, B \subset X$ is defined as follows.

$$
R(A, B)^{-1}=\inf \left\{\mathcal{E}(f, f): f \in \mathbb{R}^{\Gamma},\left.f\right|_{A}=1,\left.f\right|_{B}=0\right\} .
$$

(6) We say $(\Gamma, \mu)$ satisfies $(P H I(\beta))$, a parabolic Harnack inequality of order $\beta$ if whenever $u(n, x) \geq 0$ is defined on $[0,4 N] \times \bar{B}(y, 2 R)$ and satisfies

$$
u(n+1, x)-u(n, x)=\mathcal{L} u(n, x), \quad(n, x) \in[0,4 N] \times B(y, 2 R),
$$

then

$$
\max _{\substack{N \leq n \leq 2 N \\ x \in B(y, R)}} u(n, x) \leq C_{1} \min _{\substack{3 N \leq n \leq 4 N \\ x \in B(y, R)}}(u(n, x)+u(n+1, x)),
$$

where $N \geq 2 R$ and $N \asymp R^{\beta}$.

Theorem $2.1([25])$. Let $(\Gamma, \mu)$ be a weighted graph satisfying the $\left(p_{0}\right)$ condition. Then the following are equivalent.

(a) $(\Gamma, \mu)$ satisfies $\operatorname{PHI}(\beta)$. 
(b) $(\Gamma, \mu)$ satisfies $(V D),(E H I)$ and $\left(E_{\beta}\right)$.

(c) For $x, y \in \Gamma, n \geq d(x, y)$, the transition density of $\left\{X_{n}\right\}_{n}$ on $(\Gamma, \mu)$ satisfies

$$
\begin{gathered}
p_{n}(x, y) \leq \frac{C_{1}}{V\left(x, n^{1 / \beta}\right)} \exp \left[-\left(\frac{d(x, y)^{\beta}}{C_{1} n}\right)^{1 /(\beta-1)}\right], \\
p_{n}(x, y)+p_{n+1}(x, y) \geq \frac{C_{2}}{V\left(x, n^{1 / \beta}\right)} \exp \left[-\left(\frac{d(x, y)^{\beta}}{C_{2} n}\right)^{1 /(\beta-1)}\right] .
\end{gathered}
$$

(d) $(\Gamma, \mu)$ satisfies $(V D),(E H I)$ and $\left(R_{\beta}\right)$.

Remark 1. 1) The graphs of pre-Sierpinski gaskets, pre-Sierpinski carpets and pre-nested fractals satisfy the above conditions (cf. [4, 9, 28, 31]).

2) When the above conditions hold, then $\beta \geq 2$ (see [3] Lemma 1.1).

These equivalence conditions are very useful. But it is not clear whether these conditions are stable under bounded perturbation of forms or not. (Especially, it is still a big open problem whether $(E H I)$ is stable under bounded perturbation or not (cf. [5, 48])).

Recently, equivalent conditions to the parabolic Harnack inequality which are stable under bounded perturbation are given by Barlow-Bass [8].

Definition 2.2. $\quad(1)$ We say $(\Gamma, \mu)$ satisfies $(P I(\beta))$, a scaled Poincaré inequality with parameter $\beta \geq 2$, if there exists a constants $C_{1}>0$ such that for any ball $B=B\left(x_{0}, R\right) \subset \Gamma$ with $R \geq 1$ and $f: B \rightarrow \mathbb{R}$,

$$
\sum_{x \in B}\left(f(x)-\bar{f}_{B}\right)^{2} \mu_{x} \leq C_{1} R^{\beta} \sum_{x, y \in B} \mu_{x y}(f(x)-f(y))^{2},
$$

where $\bar{f}_{B}=\mu(B)^{-1} \sum_{y \in B} f(y) \mu_{y}$.

(2) Let $\beta \geq 2$ and $\theta \in(0,1]$. We say $(\Gamma, \mu)$ satisfies $(C S(\beta, \theta))$, a cut-off Sobolev inequality with exponents $\beta$ and $\theta$, if there exist constants $C_{1}, C_{2}>0$ such that for every $x_{0} \in \Gamma, R \geq 1$, there exists a cut-off function $\varphi\left(=\varphi_{x_{0}, R}\right)$ satisfying the following properties.

(a) $\varphi(x) \geq 1$ for $x \in B\left(x_{0}, R / 2\right)$.

(b) $\varphi(x)=0$ for $x \in B\left(x_{0}, R\right)^{c}$.

(c) $|\varphi(x)-\varphi(y)| \leq C_{1}(d(x, y) / R)^{\theta}$ for all $x, y \in \Gamma$.

(d) For any ball $B\left(x_{0}, s\right)$ with $1 \leq s \leq R$ and $f: B\left(x_{0}, 2 s\right) \rightarrow \mathbb{R}$,

$$
\begin{aligned}
& \sum_{x \in B\left(x_{0}, s\right)} f(x)^{2} \sum_{y \in \Gamma} \mu_{x y}|\varphi(x)-\varphi(y)|^{2} \\
\leq & C_{2}(s / R)^{2 \theta}\left(\sum_{x, y \in B\left(x_{0}, 2 s\right)} \mu_{x y}|f(x)-f(y)|^{2}+s^{-\beta} \sum_{y \in B\left(x_{0}, 2 s\right)} f(y)^{2} \mu_{y}\right) .
\end{aligned}
$$


Theorem $2.2([8])$. Let $(\Gamma, \mu)$ be a weighted graph satisfying the $\left(p_{0}\right)$ condition. Then the following are equivalent.

(1) There exists $\theta \in(0,1]$ such that $(\Gamma, \mu)$ satisfies $(V D),(P I(\beta))$ and $(C S(\beta, \theta))$.

(2) $(\Gamma, \mu)$ satisfies $(P H I(\beta))$.

Remark 2. Note that $(C S(2,1))$ always holds. Indeed, essentially one can take $\varphi(x)=2 d\left(x, B\left(x_{0}, R\right)^{c}\right) / R$, then $|\varphi(x)-\varphi(y)| \leq 2 / R$ if $\mu_{x y}>0$, and (2.6) follows easily. Thus Theorem 2.2 is a nice extension of (1.3) to the cases of $\beta>2$.

Clearly, $(V D),(P I(\beta))$ and $(C S(\beta, \theta))$ are stable under bounded perturbation of Dirichlet forms. Moreover, the conditions are stable under rough isometries, as we will discuss in the next subsection.

On the other hand, in general it is not easy to check $(C S(\beta, \theta))$. Very recently, simpler equivalent conditions are given by Barlow-Coulhon-Kumagai [12] under a stronger volume growth condition $((V G(\beta))$ in Definition 2.3).

In order to state the results, we define the effective resistance between $x \neq y \in \Gamma$, which is a special case of (2.3), as follows.

$$
R(x, y)^{-1}=\inf \left\{\mathcal{E}(f, f): f \in \mathbb{R}^{\Gamma}, f(x)=1, f(y)=0\right\} .
$$

We define $R(x, x)=0$ for each $x \in \Gamma$. For any $x \neq y \in \Gamma, R(x, y)$ is positive and finite (see Proposition 3.1). It can be shown that $R(\cdot, \cdot)$ is a metric on $\Gamma$.

Definition 2.3. (1) We say $(\Gamma, \mu)$ satisfies a volume growth condition $(V G(\beta))$ if there exist $K>1, C_{1}>0$ with $\alpha=\log C_{1} / \log K<\beta$ such that

$$
V(x, K R) \leq C_{1} V(x, R), \quad x \in \Gamma, \quad R \geq 1 .
$$

(2) We say $(\Gamma, \mu)$ satisfies a resistance upper and lower bound of order $\beta$ $(R U E(\beta)),(R L E(\beta))$ if there exists $C_{1}, C_{2}>0$ such that for all $x, y \in \Gamma$,

$$
\begin{aligned}
& R(x, y) \leq C_{1} \frac{d(x, y)^{\beta}}{V(x, d(x, y))}, \\
& R(x, y) \geq C_{2} \frac{d(x, y)^{\beta}}{V(x, d(x, y))} .
\end{aligned}
$$

Note that $(V G(\beta))$ is stronger than $(V D)$ and implies, for $\theta>1$

$$
V(x, \theta R) \leq c_{1} \theta^{\alpha} V(x, R) .
$$


Theorem 2.3 ([12]). $\quad$ Let $(\Gamma, \mu)$ be a weighted graph satisfying the $\left(p_{0}\right)$ condition and assume $(V G(\beta))$. Then the following are equivalent.

(1) $(\Gamma, \mu)$ satisfies $(U E(\beta))$ and $(L E(\beta))$.

(2) $(\Gamma, \mu)$ satisfies $(R U E(\beta))$ and $(R L E(\beta))$.

When the above conditions hold, then the Markov chain is recurrent.

In the next section, we will discuss a resistance form version (see Definition 3.1 ) of this theorem using the resistance metric directly.

We note that measure metric space versions of $[8,24,25]$ are being prepared $[6,10,26]$. There is also a paper which mentions relations between two-sided heat kernel estimates and parabolic Harnack inequalities on general measure metric spaces [30].

\section{$\S 2.3$. Stability under rough isometries}

In this subsection, we will discuss stability of parabolic Harnack inequalities under rough isometries.

Definition 2.4. $\quad$ Let $\left(\Gamma^{(1)}, \mu^{(1)}\right),\left(\Gamma^{(2)}, \mu^{(2)}\right)$ be weighted graphs satisfying the $\left(p_{0}\right)$ condition.

(1) A map $T: \Gamma^{(1)} \rightarrow \Gamma^{(2)}$ is called a rough isometry if the following holds.

There exist positive constants $a, c>1, b>0$ and $M>0$ such that

$$
\begin{aligned}
a^{-1} d^{(1)}(x, y)-b \leq d^{(2)}(T(x), T(y)) \leq a d^{(1)}(x, y)+b & \forall x, y \in \Gamma^{(1)} \\
d^{(2)}\left(T\left(\Gamma^{(1)}\right), y^{\prime}\right) \leq M & \forall y^{\prime} \in \Gamma^{(2)} \\
c^{-1} \mu_{x}^{(1)} \leq \mu_{T(x)}^{(2)} \leq c \mu_{x}^{(1)} & \forall x \in \Gamma^{(1)}
\end{aligned}
$$

where $\mu^{(i)}$ and $d^{(i)}(\cdot, \cdot)$ are the measure and the graph distance of $\left(\Gamma^{(i)}, \mu^{(i)}\right)$ respectively for $i=1,2$.

(2) $\left(\Gamma^{(1)}, \mu^{(1)}\right),\left(\Gamma^{(2)}, \mu^{(2)}\right)$ are said to be roughly isometric if there is a rough isometry between them.

The notion of rough isometry was first introduced by M. Kanai $([32,33])$. As the work was mainly concerned with Riemannian manifolds, the definition of the rough isometry included only (2.8), (2.9). A definition equivalent to Definition 2.4 is given in [17].

$\operatorname{PHI}(\beta)$ is stable under rough isometries.

Theorem $2.4([28])$. Let $\left(\Gamma^{(1)}, \mu^{(1)}\right), \quad\left(\Gamma^{(2)}, \mu^{(2)}\right)$ be weighted graphs satisfying the $\left(p_{0}\right)$ condition. If $\left(\Gamma^{(1)}, \mu^{(1)}\right)$ satisfies $(P H I(\beta))$ with respect to 
the graph distance and $\left(\Gamma^{(1)}, \mu^{(1)}\right),\left(\Gamma^{(2)}, \mu^{(2)}\right)$ are roughly isometric, then $\left(\Gamma^{(2)}\right.$, $\left.\mu^{(2)}\right)$ also satisfies $(P H I(\beta))$ with respect to the graph distance.

\section{§3. Results on Resistance Forms}

In this section, we will discuss a resistance form version of Theorem 2.3. We use the resistance metric directly and the time scale function is generalized from $r^{\beta}$ to $h(r)=r V(r)$ for some increasing function $V$ with doubling property.

We first define resistance forms, introduced by Kigami in [34, 35].

Definition 3.1. $\quad$ Let $X$ be a set. A pair $(\mathcal{E}, \hat{\mathcal{F}})$ is called a resistance form on $X$ if it satisfies the following.

$(1) \mathcal{E}$ is a non-negative symmetric quadratic form and $\hat{\mathcal{F}}:=\left\{f \in \mathbb{R}^{X}: \mathcal{E}(f, f)<\right.$ $\infty\} . \mathcal{E}(u, u)=0$ if and only if $u$ is constant on $X$.

(2) Let $\sim$ be an equivalent relation on $\hat{\mathcal{F}}$ defined by $u \sim v$ if and only if $u-v$ is constant on $X$. Then, $(\hat{\mathcal{F}} / \sim, \mathcal{E})$ is a Hilbert space.

(3) For any finite subset $V \subset X$ and for any $v \in \mathbb{R}^{V}$, there exists $u \in \hat{\mathcal{F}}$ such that $\left.u\right|_{V}=v$.

(4) For any $p, q \in X$,

$$
\sup \left\{\frac{|u(p)-u(q)|^{2}}{\mathcal{E}(u, u)}: u \in \hat{\mathcal{F}}, \mathcal{E}(u, u)>0\right\}<\infty .
$$

(5) (Markov property): If $u \in \hat{\mathcal{F}}$, then $\bar{u} \in \hat{\mathcal{F}}$ and $\mathcal{E}(\bar{u}, \bar{u}) \leq \mathcal{E}(u, u)$, where $\bar{u}:=(0 \vee u) \wedge 1$.

Note that (1) is slightly modified from the original definition in [34, 35].

Electric networks of weighted graphs are resistance forms.

Proposition 3.1. $\quad$ Let $(\Gamma, \mu)$ be a weighted graph satisfying the $\left(p_{0}\right)$ condition. Define $\mathcal{E}$ as in $(2.1)$ and $\hat{\mathcal{F}}:=\left\{f \in \mathbb{R}^{\Gamma}: \mathcal{E}(f, f)<\infty\right\}$. Then $(\mathcal{E}, \hat{\mathcal{F}})$ is a resistance form.

Proof. (1), (2), (3), (5) of Definition 3.1 are easy by definition of the weighted graph. So, we will prove (4). Denote the left hand side of (3.1) as $M(p, q)$. Note that, for each $u \in \hat{\mathcal{F}}$ which is not a constant function, we can take $\alpha, \beta \in \mathbb{R}$ so that $v=\alpha u+\beta$ satisfies $v(p)=1, v(q)=0$. Thus

$$
\begin{aligned}
M(p, q) & =\sup \left\{\mathcal{E}(v, v)^{-1}: v \in \hat{\mathcal{F}}, v(p)=1, v(q)=0\right\} \\
& =(\inf \{\mathcal{E}(v, v): v \in \hat{\mathcal{F}}, v(p)=1, v(q)=0\})^{-1}
\end{aligned}
$$


Now take a shortest step $p=x_{0}, x_{1}, \ldots, x_{n-1}, x_{n}=q$ such that $x_{i} \sim x_{i+1}$ $(0 \leq i \leq n-1)$. Then we have

$$
M(p, q)^{-1} \geq \inf \left\{\frac{1}{2} \sum_{i=0}^{n-1}\left(v\left(x_{i}\right)-v\left(x_{i+1}\right)\right)^{2} \mu_{x_{i} x_{i+1}}: v(x)=1, v(y)=0\right\} .
$$

Using the $\left(p_{0}\right)$ condition and Jensen's inequality, the right hand side is greater than or equal to $c_{1} / n>0$ for some $c_{1}>0$.

We note that we can also prove $M(p, q)>0$ for $p \neq q$. Indeed, by taking $h \in \mathbb{R}^{\Gamma}$ so that $h(p)=1$ and $h(z)=0$ for all $z \neq p$, we have $M(p, q)^{-1} \leq$ $\sum_{y: y \sim p} \mu_{p y} / 2=\mu_{p} / 2<\infty$.

Let $(\mathcal{E}, \hat{\mathcal{F}})$ be a resistance form on a set $X$. We define the effective resistance between points by the left hand side of (3.1) (which is equal to (2.7), with $f \in \hat{\mathcal{F}}$ instead of $f \in \mathbb{R}^{\Gamma}$ ). Then the following is proved in section 2.3 of [35].

Lemma 3.1. 1) The function $R(\cdot, \cdot)$ is a metric on $X$.

2) For all $f \in L^{2}(X, \mu)$ and $x, y \in X$,

$$
|f(x)-f(y)|^{2} \leq R(x, y) \mathcal{E}(f, f) .
$$

Further, for each $x, y \in X$, there exists $f$ so that the equality holds in (3.2).

Using this lemma, we may take a completion of $X$. Then, one can prove that $(\mathcal{E}, \hat{\mathcal{F}})$ is a resistance form on the completion of $X$ and the resistance metric can be naturally extended (see Theorem 2.3.10 in [35]). Thus, we may and will assume that $(X, R)$ is complete.

We note the definition of $R$ can be generalized for arbitrary disjoint $A, B \subset$ $X$ as in (2.3), with $f \in \hat{\mathcal{F}}$ instead of $f \in \mathbb{R}^{\Gamma}$.

Now, let $(X, d, \mu)$ be a measure metric space. Throughout the paper, we assume that $X$ is a locally compact separable connected metric space, $\mu$ is a non-negative $\sigma$-finite Borel measure on $X$, finite on compact sets and strictly positive on non-empty open sets and $d$ is a metric on $X$. We assume $(\mathcal{E}, \hat{\mathcal{F}})$ is a resistance form on $X$ and the topology induced by the resistance metric is compatible with the topology induced by $d$. Further, we assume that $\mathcal{E}$ is local, i.e. for each $u, v \in \hat{\mathcal{F}}$ where Supp $u$ and Supp $v$ are disjoint compact sets, $\mathcal{E}(u, v)=0$. Then, $\hat{\mathcal{F}} \cap C_{0}(X)$ is closable where $C_{0}(X)$ is a space of compact supported continuous functions on $X$ with respect to $d$. Denoting its $\mathcal{E}_{1}$-closure as $\mathcal{F}$, we can prove that $(\mathcal{E}, \mathcal{F})$ is a local regular Dirichlet form on $L^{2}(X, \mu)$ (see [22] for details on Dirichlet forms). We denote $-\mathcal{L}$ the corresponding non-negative self-adjoint operator. Note that due to (3.2), elements in $\mathcal{F}$ are 
Hölder continuous with respect to the resistance metric. Since the topology induced by $d$ and by the resistance metric are compatible, $\mathcal{F} \subset C(X)$. Thus the corresponding diffusion is determined without any ambiguity of starting points and the corresponding semigroup $P_{t}$ is Feller, i.e. $P_{t}\left(C_{b}(X)\right) \subset C_{b}(X)$. Using the fact $\mathcal{F} \subset C(X)$, we can also prove that there exists a version of the transition density (heat kernel) $p_{t}(x, y)$ for each $(t, x, y) \in(0, \infty) \times X \times X$.

From now on, $B(x, r)$ and $V(x, r)$ will be defined with respect to the resistance metric as follows; let $\hat{B}(x, r):=\{y \in X: R(x, y)<r\}$ and $B(x, r)$ be the connected component of $\hat{B}(x, r)$ containing $x, V(x, r):=\mu(B(x, r))$. Note that $\hat{B}(x, r)$ is not necessarily connected (see Remark 7.19 of [2]).

Throughout this paper, let $V:\left[0, R_{X}\right) \rightarrow[0, \infty)$ be a strictly increasing function with $V(0)=0$ and $V(2 r) \leq C_{1} V(r)$ for all $r \in\left[0, R_{X}\right)$, where $R_{X}$ is the diameter of $X$ with respect to the resistance metric. Note that $V(r)$ is different from $V(x, r)$. We set $h(r):=r V(r)$. $V$ will be used as a volume growth function and $h$ as a time scale function.

We now give several notions which will be used.

- Uniform volume growth with volume doubling condition: there exist $C_{1}, C_{2}>0$ such that

$$
C_{1} V(r) \leq V(x, r) \leq C_{2} V(r) \quad \text { for all } x \in X, r \in\left[0, R_{X}\right) .
$$

Remark 3. 1) When $(\Gamma, \mu)$ is a weighted graph satisfying the $\left(p_{0}\right)$ condition, $\left(\mathcal{E}, \hat{\mathcal{F}} \cap L^{2}(X, \mu)\right)$ is a local regular Dirichlet form on $L^{2}(X, \mu)$, where $(\mathcal{E}, \hat{\mathcal{F}})$ is given in Proposition 3.1. But $\left(\mathcal{E}, \hat{\mathcal{F}} \cap L^{2}(X, \mu)\right)$ does not satisfy $(U V D)$ for very small $r$. A relevant condition for such discrete examples is that $(U V D)$ holds for $r \in\left[R_{X}^{\prime}, R_{X}\right)$ where $0 \leq R_{X}^{\prime}<R_{X}$ instead of $r \in\left[0, R_{X}\right.$ ) (we denote this modified condition as $\left.\left(U V D^{\prime}\right)\right)$.

2) The weighted graph on $\mathbb{Z}^{d}(d \geq 2)$ with $\mu_{x y} \equiv 1$ for $x \sim y$ does not satisfy $\left(U V D^{\prime}\right)$. Indeed, $V(x, r)=\infty$ if $r$ is large for $d \geq 3$, and $V(r)=\exp \left(c_{1} r\right)$ for $d=2$.

- Upper and lower heat kernel estimates: there exist $C_{1}, C_{2}>0$ such that the following holds for all $x, y \in X, t \in\left(0, T_{X}\right), r \in\left[0, R_{X}\right)$.

$$
\begin{aligned}
& p_{t}(x, y) \leq \frac{C_{1} h^{-1}(t)}{t} \exp \left(-\frac{R(x, y)}{C_{1} V^{-1}(t / R(x, y))}\right), \\
& p_{t}(x, y) \geq \frac{C_{2} h^{-1}(t)}{t} \exp \left(-\frac{R(x, y)}{C_{2} V^{-1}(t / R(x, y))}\right) .
\end{aligned}
$$

Here $T_{X}>0$ is a constant depending only on $X$, such that $T_{X}<\infty$ if $\operatorname{diam} X<$ $\infty$ and $T_{X}=\infty$ if $\operatorname{diam} X=\infty$. 
Note that $h^{-1}(t) / t=1 / V\left(h^{-1}(t)\right)$. We will sometimes express the pair of estimates $(U E(h)),(L E(h))$ as

$$
p_{t}(x, y) \simeq \frac{C_{1} h^{-1}(t)}{t} \exp \left(-\frac{R(x, y)}{C_{1} V^{-1}(t / R(x, y))}\right) .
$$

- There exist $C_{2}, C_{3}>0$ such that

$$
p_{t}(x, y) \geq \frac{C_{2} h^{-1}(t)}{t} \quad \text { if } \quad h\left(C_{3} R(x, y)\right) \leq t .
$$

- For $A \subset X$, let $T_{A}$ be the first exit time from $A$, i.e. $T_{A}=\min \{t \geq 0$ : $\left.X_{t} \notin A\right\}$. Then the following holds for all $x \in X, t \in\left(0, T_{X}\right), r \in\left[0, R_{X}\right)$.

$$
\begin{gathered}
\mathbb{E}^{x}\left[T_{B(x, r)}\right] \simeq h(r), \\
\Psi_{t}(x, r):=\mathbb{P}^{x}\left(T_{B(x, r)} \leq t\right) \leq C_{1} \exp \left(-\frac{C_{2} r}{V^{-1}(t / r)}\right) .
\end{gathered}
$$

- Parabolic Harnack inequality of order $h$ : for some $0<\gamma_{1}<\gamma_{2}<$ $\gamma_{3}<\gamma_{4}, 0<\delta<1$ and for any $s \in\left(0, T_{X}\right), r \in\left[0, R_{X}\right), x \in X$, set $Q=$ $\left(s, s+\gamma_{4} h(r)\right) \times B(x, r)$ and

$$
\begin{aligned}
& Q_{-}:=\left(s+\gamma_{1} h(r), s+\gamma_{2} h(r)\right) \times B(x, \delta r), \\
& Q_{+}:=\left(s+\gamma_{3} h(r), s+\gamma_{4} h(r)\right) \times B(x, \delta r) .
\end{aligned}
$$

Let $u(t, x) \geq 0$ be defined on $Q$ and satisfy

$$
\frac{\partial u(t, x)}{\partial t}=\mathcal{L} u(t, x), \quad(t, x) \in Q .
$$

Then,

$$
\sup _{(t, x) \in Q_{-}} u(t, x) \leq C_{1} \inf _{(t, x) \in Q_{+}} u(t, x) .
$$

Let $(X, d, \mu)$ be a measure metric space. Our main theorem is the following.

Theorem 3.1. $\quad$ Let $(\mathcal{E}, \hat{\mathcal{F}})$ be a resistance form on $X$ and assume that the topology induced by the resistance metric is compatible with the topology induced by $d$. Assume further that $\mathcal{E}$ is local and let $\mathcal{F}$ be the $\mathcal{E}_{1}$-closure of $\hat{\mathcal{F}} \cap C_{0}(X)$. Then the following are equivalent.

(1) $(\mathcal{E}, \mathcal{F})$ satisfies $(U V D)$.

$(2)(\mathcal{E}, \mathcal{F})$ satisfies $(U E(h))$ and $(N L E(h))$. 
If we further assume the following, we can obtain an equivalence to the full two-sided heat kernel estimate.

- Chain condition: there exists $C_{1}>0$ such that for all $x, y \in X$ and all $n \in \mathbb{N}$, there exists $\left\{x_{0}, x_{1}, \ldots, x_{n}\right\} \subset X$ with $x_{0}=x, x_{n}=y$ such that

$$
R\left(x_{i}, x_{i+1}\right) \leq C_{1} R(x, y) / n \quad \text { for all } 0 \leq i \leq n-1 .
$$

Corollary 3.1. Let $(\mathcal{E}, \mathcal{F})$ be as in Theorem 3.1 and assume that $(C C)$ holds. Then the following are equivalent.

(1) $(\mathcal{E}, \mathcal{F})$ satisfies $(U V D)$.

$(2)(\mathcal{E}, \mathcal{F})$ satisfies $(U E(h))$ and $(L E(h))$.

Remark 4. In fact, $(C C)$ "seldom" holds; even for the Dirichlet form corresponding to Brownian motion on the Sierpinski gasket, $(C C)$ does not hold (see Example 2 in $\S 5$ ). However, when $X$ is a tree, $(C C)$ holds (Example 1 in $§ 5)$.

Under $(U V D)$, we always have $(P H I(h))$.

Proposition 3.2. Let $(\mathcal{E}, \mathcal{F})$ be as in Theorem 3.1 and assume that $(U V D)$ holds. Then, $(\mathcal{E}, \mathcal{F})$ satisfies $(P H I(h))$.

Remark 5. Note that these results can be generalized (by essentially the same proof) under the volume doubling condition. Let $h^{x}(r)=r V(x, r)$. Then, $(V D)$ implies $\left(U E\left(h^{x}\right)\right),\left(N L E\left(h^{x}\right)\right)$ and $\left(P H I\left(h^{x}\right)\right)$ for all $x \in X$. Assume in addition $(C C)$, then $\left(L E\left(h^{x}\right)\right)$ holds for all $x \in X$.

Finally, we note that $(P H I(h))$ implies slightly weaker heat kernel estimates. The next proposition is a modification of a result in [30]. Here we do not need to assume the Dirichlet form to be a resistance form.

Proposition 3.3. Let $(\mathcal{E}, \mathcal{F})$ be a local regular Dirichlet form on $L^{2}(X, \mu)$ and assume that $(P H I(h))$ holds for some $h: \mathbb{R}_{+} \rightarrow \mathbb{R}_{+}$where $V(r):=h(r) / r$ is an increasing function with $V(2 r) \leq C_{1} V(r)$ for all $r \in$ $\left[0, R_{X}\right)$. Then, there exists a heat kernel of $(\mathcal{E}, \mathcal{F})$ with the following estimates.

$$
\begin{aligned}
& p_{t}(x, y) \leq \frac{C_{1}}{V\left(x, h^{-1}(t)\right)} \exp \left(-\frac{R(x, y)}{C_{1} V^{-1}(t / R(x, y))}\right), \\
& p_{t}(x, y) \geq \frac{C_{2}}{V\left(x, h^{-1}(t)\right)} \quad \text { for } \quad h\left(C_{3} R(x, y)\right) \leq t .
\end{aligned}
$$


Remark 6. As mentioned in Remark 3, discrete examples such as weighted graphs do not satisfy $(U V D)$ so the assertions here do not include them. By modifying the conditions (for example $\left(U V D^{\prime}\right)$ instead of $(U V D)$ ), we may give statements that include such cases, but we do not discuss them here.

\section{§4. Proof}

In this section, we will give the proof of the results in section 3 . We note that most of the proof in $\S 4.1$ and $\S 4.2$ is based on the proof in [12].

\section{§4.1. Proof of theorem $3.1(1) \Rightarrow(2)$ : upper bound}

We begin with the on-diagonal upper bound.

Proposition 4.1. Assume $(U V D)$. Then, there exists $C_{1}>0$ such that

$$
p_{t}(x, y) \leq \frac{C_{1} h^{-1}(t)}{t}, \quad \text { for all } x \in X, t \in\left(0, T_{X}\right) .
$$

Proof. We follow the proof in [12]. Let $f_{t}(y)=p_{t}(x, y)$ and

$$
\psi(t)=\left\|f_{t}\right\|_{2}^{2}=p_{2 t}(x, x)=f_{2 t}(x) .
$$

Since $\int_{B(x, r)} f_{t} d \mu \leq 1$ for $r>0$, there exists $y=y(t, r) \in B(x, r)$ with $f_{t}(y) \leq$ $V(x, r)^{-1} \leq c_{1} V(r)^{-1}$. Using (3.2),

$$
\frac{1}{2} f_{t}(x)^{2} \leq f_{t}(y)^{2}+\left|f_{t}(x)-f_{t}(y)\right|^{2} \leq \frac{c_{1}^{2}}{V(r)^{2}}+\mathcal{E}\left(f_{t}, f_{t}\right) R(x, y) .
$$

Since $R(x, y)<r$, it follows that

$$
r \mathcal{E}\left(f_{t}, f_{t}\right) \geq \frac{1}{2} \psi(t / 2)^{2}-\frac{c_{1}^{2}}{V(r)^{2}} .
$$

Hence

$$
\psi^{\prime}(t)=-2 \mathcal{E}\left(f_{t}, f_{t}\right) \leq \frac{2 c_{1}^{2} V(r)^{-2}-\psi(t / 2)^{2}}{r} .
$$

Now $\psi^{\prime \prime}(t)=4\left(\mathcal{L} f_{t}, \mathcal{L} f_{t}\right) \geq 0$, so $\psi^{\prime}(t / 2) \leq \psi^{\prime}(t)$. We thus obtain

$$
\psi^{\prime}(t) \leq \psi^{\prime}(2 t) \leq \frac{2 c_{1}^{2} V(r)^{-2}-\psi(t)^{2}}{r} .
$$

Set $\varphi(t)=2 / \psi(t)$, then $\varphi$ is increasing and

$$
\varphi^{\prime}(t)=-\frac{1}{2} \varphi(t)^{2} \psi^{\prime}(t) \geq \frac{2-c_{1}^{2} \varphi(t)^{2} V(r)^{-2}}{r} .
$$


So, if $r=r(t)$ is chosen so that $\varphi(t) \leq V(r(t)) / c_{1}$, then $\varphi^{\prime}(t) \geq r(t)^{-1}$.

Choose $r$ so that $V(r(t))=c_{1} \varphi(t)$; then $r$ is increasing. Hence $r^{\prime}(t) V^{\prime}(r(t))$ $=c_{1} \varphi^{\prime}(t) \geq c_{1} r(t)^{-1}$ and we have

$$
c_{1} t \leq \int_{0}^{t} r(s)(V(r(s)))^{\prime} d s \leq r(t) V(r(t)) .
$$

Let $R>0$, and choose $s$ so that $r(s)=R$. Then $c_{1} s \leq r(s) V(r(s))=R V(R)$, so $\varphi\left(R V(R) / c_{1}\right) \geq \varphi(s)=V(r(s)) / c_{1}=V(R) / c_{1}$. Noting that $h(R)=R V(R)$, we have $p_{2 h(R) / c_{1}}(x, x) \leq 2 c_{1} R h(R)^{-1}$. Rearranging, we obtain $(D U E(h))$.

Lemma 4.1. Under (UVD), the following holds.

$$
C_{1} r \leq R\left(x, B(x, r)^{c}\right) \leq r \quad \text { for all } r \geq 0, x \in X .
$$

Proof. Take $y, z \in B(x, r)$ with $R(y, z)=\lambda r, \lambda \leq 1$. We have by (3.2),

$$
|f(y)-f(z)|^{2} \leq R(y, z) \mathcal{E}(f, f)=\lambda r \mathcal{E}(f, f), \quad \text { for all } f \in \mathcal{F} .
$$

Let $z \in X$ be such that $c_{1} r \leq R(x, z) \leq r$ for some $c_{1}<1$. If $h_{z}$ is the harmonic function on $X \backslash\{x, z\}$ with $h_{z}(z)=0, h_{z}(x)=1$ then $\mathcal{E}\left(h_{z}, h_{z}\right)=R(x, z)^{-1}$. Applying (4.2), we have, if $R(y, z)=\lambda r$,

$$
\left|h_{z}(y)\right|^{2}=\left|h_{z}(y)-h_{z}(z)\right|^{2} \leq \frac{\lambda r}{R(x, z)} \leq \frac{\lambda}{c_{1}} .
$$

So there exists a constant $\lambda_{1}$ such that $R(y, z) \leq \lambda_{1} r$ implies that $h_{z}(y) \leq \frac{1}{2}$.

Now use $(U V D)$ to cover $B(x, r) \backslash B\left(x, c_{1} r\right)$ by balls $B\left(z_{i}, \lambda_{1} r\right), 1 \leq i \leq M$, with $c_{1} r \leq R\left(x, z_{i}\right) \leq r$. (Volume doubling condition is enough to do this and $M$ depends only on the volume doubling constant.) Let $g=\min h_{z_{i}}$, and $h=2\left(g-\frac{1}{2}\right)^{+} \cdot 1_{B(x, r)}$. Then $h(x)=1$, and $h=0$ on $B\left(x, c_{1} r\right)^{c}$, so that

$$
R\left(x, B(x, r)^{c}\right)^{-1} \leq \mathcal{E}(h, h) \leq 4 \sum_{i} \mathcal{E}\left(h_{z_{i}}, h_{z_{i}}\right) \leq 4 M\left(\min _{i} R\left(x, z_{i}\right)\right)^{-1} \leq c_{2} r^{-1} .
$$

We thus obtain the first inequality of (4.1). The second inequality of (4.1) is clear because $R\left(x, B(x, r)^{c}\right) \leq R(x, y)$ for all $y \in \partial B(x, r)$.

From this lemma, we see that $\hat{B}\left(x, C_{1} r\right) \subset B(x, r)$.

Proposition 4.2. Assume $(U V D)$. Then there exist $C_{1}, C_{2}>0$ such that the following holds for all $r \geq 0$ and $x_{0}, x \in X$.

$$
\mathbb{E}^{x_{0}}\left[T_{B\left(x_{0}, r\right)}\right] \geq C_{1} h(r), \quad \mathbb{E}^{x}\left[T_{B\left(x_{0}, r\right)}\right] \leq C_{2} h(r) .
$$

In particular, $\left(E_{h}\right)$ holds. 
Proof. Denote $B:=B\left(x_{0}, r\right)$ and let $\left(\mathcal{E}_{B}, \mathcal{F}_{B}\right)$ be the part of the Dirichlet form in the sense of [22] section 4.4. By Theorem 4.4.3 of [22], it is a regular Dirichlet form on $L^{2}(B, \mu)$ with $\mathcal{F}_{B} \subset\left\{f \in \mathcal{F}: f(x)=0\right.$ on $\left.x \in B^{c}\right\}$. Let $X_{t}^{B}$ be the corresponding Hunt process, which is a process with the killing condition outside $B$. Using (3.2), we have

$$
\sup _{x \in B}|f(x)|^{2} \leq r \mathcal{E}(f, f) \quad \text { for all } f \in \mathcal{F}_{B} .
$$

Thus, $\left(\mathcal{E}_{B}, \mathcal{F}_{B}\right)$ is a transient Dirichlet form so that the extended Dirichlet space $\left(\mathcal{E}_{B},\left(\mathcal{F}_{B}\right)_{e}\right)$ is a Hilbert space (Theorem 1.5.3 in [22]). Using (4.4) and the Riesz representation theorem, there exists a Green kernel $g_{B}(\cdot, \cdot)$ with the reproducing property; $\mathcal{E}\left(g_{B}(x, \cdot), f\right)=f(x)$ for all $f \in \mathcal{F}_{B}$. Using the reproducing property and Definition $3.1(1), g_{B}(x, y)=g_{B}(y, x)$ and $g_{B}(x, x)>0$ for all $x, y \in B$. Set $p_{x}(y):=g_{B}(x, y) / g_{B}(x, x)$. Then $p_{x}$ is an equilibrium potential for $R\left(x, B^{c}\right)$ and we have

$$
R\left(x, B^{c}\right)^{-1}=\mathcal{E}\left(p_{x}, p_{x}\right)=g_{B}(x, x)^{-1} .
$$

Since $p_{x}(y) \leq 1$ for all $y \in X$,

$$
g_{B}(x, y) \leq g_{B}(x, x) \quad \text { for all } x, y \in X .
$$

On the other hand, by the definition of the resistance,

$$
R\left(x, B^{c}\right) \leq R(x, y) \quad \text { for all } x, y \in X, y \in B^{c},
$$

so that $g_{B}(x, x) \leq r$. Now, since

$$
\mathbb{E}^{x}\left[T_{B\left(x_{0}, r\right)}\right]=\int_{B} g_{B}(x, y) d \mu(y),
$$

we have

$$
\mathbb{E}^{x}\left[T_{B\left(x_{0}, r\right)}\right] \leq r V\left(x_{0}, r\right) \leq c_{1} h(r),
$$

where we use (4.6) and $(U V D)$. We thus obtain the second inequality of (4.3).

Next, by (3.2) and the reproducing property of $g_{B}$, we have for $y \in B$,

$$
\left|g_{B}\left(x_{0}, x_{0}\right)-g_{B}\left(x_{0}, y\right)\right|^{2} \leq \mathcal{E}\left(g_{B}, g_{B}\right) R\left(x_{0}, y\right)=g_{B}\left(x_{0}, x_{0}\right) R\left(x_{0}, y\right) .
$$

Thus, by (4.5) we have

$$
\left|1-p_{x_{0}}(y)\right|^{2} \leq \frac{R\left(x_{0}, y\right)}{R\left(x_{0}, B^{c}\right)} .
$$


Now using Lemma 4.1, we see that there exists $\delta>0$ such that

$$
p_{x_{0}}(y)=\frac{g_{B}\left(x_{0}, y\right)}{g_{B}\left(x_{0}, x_{0}\right)} \geq 1 / 2 \quad \text { for all } y \in B\left(x_{0}, \delta r\right) .
$$

On the other hand, by (4.5) and Lemma 4.1, we have $g_{B}\left(x_{0}, x_{0}\right)=R\left(x_{0}, B^{c}\right) \geq$ $c_{2} r$. Combining this with (4.8), we have

$$
g_{B}\left(x_{0}, y\right) \geq c_{3} r, \quad \text { for all } y \in B\left(x_{0}, \delta r\right) .
$$

Using this with (4.7) and (UVD), we have

$$
\mathbb{E}^{x_{0}}\left[T_{B\left(x_{0}, r\right)}\right]=\int_{B} g_{B}\left(x_{0}, y\right) d \mu(y) \geq c_{4} r V\left(x_{0}, \delta r\right) \geq c_{5} h(r),
$$

where $c_{5}>0$ depends on $\delta$. We thus obtain the first inequality of (4.3).

The next lemma is known when $h(r)=r^{\beta}$ for some $\beta \geq 2$. The following proof is a modification of the known proof.

Lemma 4.2. $\quad(4.3) \Rightarrow(\Psi)$.

Proof. We first prove that there exists $0<c_{1}<1$ and $c_{2}>0$ such that

$$
\mathbb{P}^{x}\left(T_{B(x, r)} \leq s\right) \leq 1-c_{1}+c_{2} s / h(r) \quad \text { for all } x \in X, s \geq 0 .
$$

Indeed, by the Markov property, for each $x \in X$ we have

$$
\mathbb{E}^{x} T_{B(x, r)} \leq s+\mathbb{E}^{x}\left[1_{\left\{T_{B(x, r)}>s\right\}} \mathbb{E}^{X_{s}} T_{B(x, r)}\right] .
$$

Applying (4.3), we have

$$
c_{3} h(r) \leq s+c_{4} h(r) \mathbb{P}^{x}\left(T_{B(x, r)}>s\right)=s+c_{4} h(r)\left(1-\mathbb{P}^{x}\left(T_{B(x, r)} \leq s\right)\right) .
$$

Rearranging gives (4.9).

Next, let $l \geq 1, b=r / l$, and define stopping times $\sigma_{i}, i \geq 0$ by

$$
\sigma_{0}=0, \quad \sigma_{i+1}=\inf \left\{t \geq \sigma_{i}: R\left(X_{\sigma_{i}}, X_{t}\right) \geq b\right\} .
$$

Let $\xi_{i}=\sigma_{i}-\sigma_{i-1}, i \geq 1$. Let $\mathcal{F}_{t}$ be the filtration generated by $\left\{X_{s}: s \leq t\right\}$ and let $\mathcal{G}_{m}=\mathcal{F}_{\sigma_{m}}$. We have by (4.9)

$$
\mathbb{P}^{x}\left(\xi_{i+1} \leq t \mid \mathcal{G}_{i}\right)=\mathbb{P}^{X_{\sigma_{i}}}\left(T_{B\left(X_{\sigma_{i}}, b\right)} \leq t\right) \leq p+c_{2} t / h(b),
$$


where $0<p<1$. As $R\left(X_{\sigma_{i}}, X_{\sigma_{i+1}}\right)=b$, we have $R\left(X_{0}, X_{\sigma_{l}}\right) \leq r$, so that $\sigma_{l}=\sum_{i=1}^{l} \xi_{i} \leq T_{B\left(X_{0}, r\right)}$. So, by Lemma 3.14 in [2],

$\log \mathbb{P}^{x}\left(T_{B(x, r)} \leq t\right) \leq 2 p^{-1 / 2}\left(\frac{c_{2} l t}{h(r / l)}\right)^{1 / 2}-l \log (1 / p)=c_{5}\left(\frac{l t}{h(r / l)}\right)^{1 / 2}-c_{6} l$.

Now take $l_{0} \in \mathbb{N}$ the largest integer $l$ that satisfies

$$
c_{6} l / 2>c_{5}\left(\frac{l t}{h(r / l)}\right)^{1 / 2} .
$$

This is equivalent to $r / l>V^{-1}\left(c_{7} t / r\right)$ where $c_{7}=4 c_{5}^{2} / c_{6}^{2}$. Note that if $r \leq$ $V^{-1}\left(c_{7} t / r\right)$, then $(\Psi)$ clearly holds by taking $C_{1}>0$ large, so we may assume that (4.10) holds for small $l \in \mathbb{N}$. Then

$$
l_{0}<\frac{r}{V^{-1}\left(c_{7} t / r\right)} \leq l_{0}+1, \quad \text { and } \quad \log \mathbb{P}^{x}\left(T_{B(x, r)} \leq n\right) \leq-c_{6} l_{0} / 2 .
$$

We thus obtain $(\Psi)$.

Remark 7. When $h(r)=r^{\beta},(\Psi)$ turns out to be the following.

$$
\mathbb{P}^{x}\left(T_{B(x, r)} \leq t\right) \leq C \exp \left(-c\left(r^{\beta} / t\right)^{1 /(\beta-1)}\right) .
$$

In order to show $(U E(h))$, all we need now is to prove $(U V D)+(D U E(h))+$ $(\Psi) \Rightarrow(U E(h))$. Since the proof is standard, we omit it (see, for example, Theorem 3.11 in $[2])$.

\section{§4.2. $\quad$ Proof of theorem $3.1(1) \Rightarrow(2)$ : lower bound}

We first give an upper estimate for the time derivative of the heat kernel, which will be used later. Since it is well-known, we omit the proof (see, for example, Proposition 4.16 in [2]).

Lemma 4.3. Assume that $(U V D)$ and $(D U E(h))$ holds. Then,

$$
\left|\frac{\partial_{t}}{\partial t} p_{t}(x, x)\right|=\mathcal{E}\left(p_{t / 2}(\cdot, x), p_{t / 2}(\cdot, x)\right) \leq \frac{C_{1} h^{-1}(t)}{t^{2}}, \quad \forall x, y \in X, t>0 .
$$

We now start proving $(N L E(h))$. To begin with, we will obtain the ondiagonal lower estimate.

Proposition 4.3. Assume $(U V D)$ and $\left(E_{h}\right)$. Then, there exists $C_{1}>0$ such that for all $t>0$,

$$
p_{t}(x, x) \geq \frac{C_{1} h^{-1}(t)}{t} .
$$


Proof. The proof is standard. Using (4.9) we have that

$$
\mathbb{P}^{x}\left(X_{t} \notin B(x, r)\right) \leq \mathbb{P}\left(T_{B(x, r)} \leq t\right) \leq 1-c_{1}+c_{2} t / h(r) .
$$

Hence by choosing $r$ such that $c_{3} h(r)<t<c_{1} h(r) /\left(2 c_{2}\right)$ for some $c_{3}>0$, we have

$$
\mathbb{P}^{x}\left(X_{t} \notin B(x, r)\right) \leq c_{4}<1 .
$$

Thus $\mathbb{P}^{x}\left(X_{t} \in B(x, r)\right) \geq 1-c_{4}>0$. By Cauchy-Schwarz,

$$
\left(1-c_{4}\right)^{2} \leq \mathbb{P}^{x}\left(X_{t} \in B(x, r)\right)^{2}=\left(\int_{B(x, r)} p_{t}(x, z) d \mu(z)\right)^{2} \leq V(x, r) p_{2 t}(x, x) .
$$

Now, using the lower bound on our choice of $t$ and $(U V D)$, we obtain

$$
p_{2 t}(x, x) \geq \frac{c_{5} h^{-1}(t)}{t} .
$$

Rewriting in terms of $t$ and adjusting, we have the result.

We next prove a near-diagonal lower estimate.

Lemma 4.4. Assume $(U V D),(D U E(h))$ and $(D L E(h))$. Then, there exist $C_{2}, C_{3}>0$ such that

$$
p_{t}(x, y) \geq \frac{C_{2} h^{-1}(t)}{t} \quad \text { if } \quad h\left(C_{3} R(x, y)\right) \leq t .
$$

Proof. First, using (3.2) and Lemma 4.3, we have

$$
\left|p_{t}(x, y)-p_{t}\left(x, y^{\prime}\right)\right|^{2} \leq R\left(y, y^{\prime}\right) \mathcal{E}\left(p_{t}(\cdot, x), p_{t}(\cdot, x)\right) \leq \frac{c_{1} h^{-1}(t)}{t^{2}} R\left(y, y^{\prime}\right) .
$$

Using this and $(D L E(h))$,

$$
\begin{aligned}
p_{t}(x, y) & \geq p_{t}(x, x)-\left|p_{t}(x, x)-p_{t}(x, y)\right| \\
& \geq \frac{c_{2} h^{-1}(t)}{t}-\left\{\frac{c_{1} h^{-1}(t)}{t^{2}} R(x, y)\right\}^{1 / 2} \\
& =\frac{c_{2} h^{-1}(t)^{1 / 2}}{t}\left(h^{-1}(t)^{1 / 2}-c_{3} R(x, y)^{1 / 2}\right) .
\end{aligned}
$$

Now, taking $C_{3}$ large enough, we have $h^{-1}(t)^{1 / 2} / 2 \geq c_{3} R(x, y)^{1 / 2}$ if $h\left(C_{3}\right.$ $R(x, y)) \leq t$ holds. We thus obtain the result. 


\section{§4.3. Proof of the rest}

The rest of the proof in Theorem 3.1 and the proof of Corollary 3.1 is standard. So we will just mention references.

The proof $(2) \Rightarrow(1)$ in Theorem 3.1 goes by simple modifications of [24] Proposition 15.1. Under $(C C)$, we can apply the standard chain argument to deduce $(L E(h))$ from $(N L E(h))$ and $(U V D)$ (see, for example, Theorem 3.11 in [2]). Thus Corollary 3.1 holds.

For the proof of Proposition 3.2, the following lemma is important.

Lemma 4.5. Let $x_{0} \in X, B:=B\left(x_{0}, r\right)$ and let $p_{t}^{B}(x, y)$ be the transition density of the process killed at $B^{c}$. Suppose $(U E(h))$ and $(N L E(h))$ hold. Then, there exist $C_{1}, C_{2}, C_{3}>0$ and $0<\delta<1$ such that the following holds.

$p_{t}^{B}(x, y) \geq \frac{C_{1} h^{-1}(t)}{t}\left(\geq \frac{C_{2}}{V(\delta r)}\right) \forall x, y \in B\left(x_{0}, \delta r\right), h\left(C_{3} \delta r\right) \leq \forall t \leq h\left(2 C_{3} \delta r\right)$.

Proof. We will use the following Dynkin-Hunt formula,

$$
p_{t}^{B}(x, y)=p_{t}(x, y)-\mathbb{E}^{x}\left[1_{\left\{T_{B} \leq t\right\}} p_{t-T_{B}}\left(X_{T_{B}}, y\right)\right] .
$$

First, by taking $C_{3}$ as $2 C_{3}$ in Lemma 4.4 , we can estimate $p_{t}(x, y)$ from below using $(N L E(h))$.

Denote $\delta^{\prime}=C_{3} \delta$. If $\xi \in \partial B$ and $t^{\prime} \leq t$, then $R(\xi, y) \geq(1-\delta) r$. Thus, taking $\epsilon=\epsilon_{r, t^{\prime}} \in(0,1]$ such that $h\left(\epsilon \delta^{\prime} r\right) \leq t^{\prime} \leq h\left(2 \epsilon \delta^{\prime} r\right)$, we have by $(U E(h))$,

$$
\begin{aligned}
p_{t^{\prime}}(\xi, y) & \leq \frac{C_{1} h^{-1}\left(t^{\prime}\right)}{t^{\prime}} \exp \left(-\left(\frac{(1-\delta) r}{C_{1} V^{-1}\left(t^{\prime} /((1-\delta) r)\right)}\right)\right) \\
& \leq \frac{C_{2} h^{-1}(t)}{2 t}\left(\frac{2 C_{1} h^{-1}\left(t^{\prime}\right) / t^{\prime}}{C_{2} h^{-1}(t) / t}\right) \exp \left(-\frac{(1-\delta) r}{C_{2} V^{-1}\left(t^{\prime} /((1-\delta) r)\right)}\right) \\
& \leq \frac{C_{2} h^{-1}(t)}{2 t} \cdot c_{3} \frac{\epsilon h\left(\delta^{\prime} r\right)}{h\left(\epsilon \delta^{\prime} r\right)} \exp \left(-\frac{(1-\delta) r}{c_{4} V^{-1}\left(\epsilon \delta^{\prime} V\left(\epsilon \delta^{\prime} r\right) /(1-\delta)\right)}\right) \\
& \leq \frac{C_{2} h^{-1}(t)}{2 t} \cdot \frac{c_{5}}{\epsilon^{\alpha-1}} \exp \left(-\frac{1-\delta}{c_{4} \epsilon \delta^{\prime}}\right) .
\end{aligned}
$$

Here we use the fact $h(2 r) \leq c_{6} h(r)$ (we then set $\alpha=\log c_{6} / \log 2$ ) and $V^{-1}\left(\epsilon \delta^{\prime} V\left(\epsilon \delta^{\prime} r\right) /(1-\delta)\right) \leq V^{-1}\left(V\left(\epsilon \delta^{\prime} r\right)\right)=\epsilon \delta^{\prime} r$ (here we take $\delta$ small so that $\left.\delta^{\prime} /(1-\delta) \leq 1\right)$ in the last inequality. By taking $\delta$ small enough, the last term is smaller than $C_{2} h^{-1}(t) /(2 t)$. Putting this and $(N L E(h))$ into (4.13), we obtain the desired estimate.

Using this lemma, the proof of Proposition 3.2 goes by simple modifications of [20] section 3. See also section 5 of [30] and section 3.3 of [19]. Proposition 
3.3 is a special case of Theorem 5.3 in [30]. Indeed, $h^{-1}$ corresponds to $\rho$ in [30] and it satisfies conditions (R1)', (R2) in the paper. $r / V^{-1}(t / r)$ corresponds to $G(t, r)$ in the paper. Note that we cannot obtain the off-diagonal lower heat kernel estimate because the resistance metric is not a geodesic in general.

\section{$\S 5 . \quad$ Examples}

In this section, we give examples where $(U E(h))$ and $(N L E(h))$ holds.

\section{Example 1: Trees}

Let $(X, d, \mu)$ be a measure metric space. $d$ is called a geodesic if for any two points $x, y \in X$, there exists a continuous map $\gamma:[0, d(x, y)] \rightarrow X$ such that $d(\gamma(s), \gamma(t))=t-s$ for all $0 \leq s \leq t \leq d(x, y)$. $(X, d, \mu)$ is called a tree if it is the geodesic measure metric space and $\{\gamma(t): 0 \leq t \leq d(x, y)\}$ is uniquely determined (independent of the choice of $\gamma$ ). In other word, tree is a loopless geodesic space.

Assume that $(X, d, \mu)$ is a tree. Then, it is a dendrite in the sense of Kigami [34]. Thus, by Theorem 5.4 in [34] there exists a resistance form $(\mathcal{E}, \hat{\mathcal{F}})$ on $X$ associated to $(X, d)$ such that $\left(\mathcal{E}, \hat{\mathcal{F}} \cap L^{2}(X, \mu)\right)$ is a local regular Dirichlet form on $L^{2}(X, \mu)$. The resistance metric for $(\mathcal{E}, \hat{\mathcal{F}})$ is equal to $d$ so that it is a geodesic. By Theorem 3.1 and Corollary 3.1, we have the following.

Proposition 5.1. Let $(X, d, \mu)$ is a tree and assume $(\mathcal{E}, \mathcal{F})$ is an associated local regular Dirichlet form on $L^{2}(X, \mu)$. Then, $(U V D)$ with respect to $d$ is equivalent to $(U E(h))+(L E(h))$ with respect to $d$.

Proposition 5.1 generalizes the result in [16], where trees with $V(r)=r$ are treated.

Example 2: P.c.f. self-similar sets

P.c.f. self-similar sets (cf. [35]) are a class of finitely ramified fractals introduced by Kigami.

Let $X$ be a compact metrizable space with $S=\{1,2, \ldots, N\}$ and continuous injections $F_{i}: X \rightarrow X(1 \leq i \leq N) . \mathcal{L}=\left(X, S,\left\{F_{s}\right\}_{s \in S}\right)$ is called a post critically finite (p.c.f.) self-similar set if $\mathcal{L}$ satisfies the following.

1) There exists continuous surjection $\pi: S^{\mathbb{N}} \rightarrow X$ such that $\pi \circ \tilde{\sigma}_{s}=F_{s} \circ \pi$ for all $s \in S$. Here $\tilde{\sigma}_{s}: S^{\mathbb{N}} \rightarrow S^{\mathbb{N}}$ is a map such that $\tilde{\sigma}_{s}\left(w_{1} w_{2} \cdots\right)=s w_{1} w_{2} \cdots$.

2) Let $P(\mathcal{L})=\cup_{n \geq 1} \sigma^{n}\left(\pi^{-1}\left(\cup_{s, t \in S, s \neq t}\left(F_{s}(X) \cap F_{t}(X)\right)\right)\right)$, where $\sigma\left(w_{1} w_{2} \cdots\right)=$ $w_{2} w_{3} \cdots$. Then $\sharp P(\mathcal{L})<\infty$.

One of the typical examples of p.c.f. self-similar sets is the Sierpinski gasket. 
The (2-dimensional) Sierpinski gasket is a unique non-void compact set $X \subset \mathbb{R}^{2}$ that satisfies $X=\cup_{i \in S} F_{i}(X)$ where $S=\{1,2,3\}$ and $F_{i}: \mathbb{R}^{2} \rightarrow \mathbb{R}^{2}$ is given by $F_{i}(\mathbf{x})=\left(\mathbf{x}+\mathbf{a}_{i}\right) / 2$ with $\mathbf{a}_{1}=(0,0), \mathbf{a}_{2}=(1,0)$ and $\mathbf{a}_{3}=(1 / 2, \sqrt{3} / 2)$.

We will consider connected p.c.f. self-similar sets. Denote $F_{w}=F_{w_{1}} \circ$ $\cdots \circ F_{w_{m}}$ for $w=w_{1} \cdots w_{m}$ and let $V_{0}=\pi(P), V_{m}=\cup_{w \in S^{m}} F_{w}\left(V_{0}\right), V_{*}=$ $\cup_{m \geq 0} V_{m}$. Let $D: \mathbb{R}^{V_{0}} \rightarrow \mathbb{R}^{V_{0}}$ be a symmetric irreducible linear map so that $D_{p p}<0, D_{p q} \geq 0, p \neq q, \sum_{q \in V_{0}} D_{p q}=0, p \in V_{0}$, and let $r=\left(r_{1}, \ldots, r_{N}\right)$ be $r_{i}>0(i \in S)$. The simplest example of $(D, r)$ for the Sierpinski gasket is $D=\left(\begin{array}{ccc}-2 & 1 & 1 \\ 1 & -2 & 1 \\ 1 & 1 & -2\end{array}\right), r=(1,1,1)$. For $w \in S^{m}$, let $R_{w}: \mathbb{R}^{V_{m}} \rightarrow \mathbb{R}^{V_{0}}$ be as $R_{w}(u)=u \circ F_{w}$ and let $r_{w}=r_{w_{1}} \cdots r_{w_{m}}$. We define a difference operator $H_{m}: \mathbb{R}^{V_{m}} \rightarrow \mathbb{R}^{V_{m}}$ as follows,

$$
H_{m}=\sum_{w \in S^{m}} r_{w}^{-1{ }^{t}} R_{w} D R_{w}=\left(\begin{array}{c}
T_{m}{ }^{t} J_{m} \\
J_{m} K_{m}
\end{array}\right)
$$

where $T_{m}: \mathbb{R}^{V_{0}} \rightarrow \mathbb{R}^{V_{0}}, J_{m}: \mathbb{R}^{V_{0}} \rightarrow \mathbb{R}^{V_{m} \backslash V_{0}}$ and $K_{m}: \mathbb{R}^{V_{m} \backslash V_{0}} \rightarrow \mathbb{R}^{V_{m} \backslash V_{0}}$. We assume the following for $(D, r)$.

Assumption. 1) There exists $\lambda>0$ such that $T_{1}-{ }^{t} J_{1} K_{1}^{-1} J_{1}=\lambda^{-1} D$.

2) $r_{s}<\lambda$ for all $s \in S$.

For $u, v \in \mathbb{R}^{V_{m}}$, define a quadratic form $\mathcal{E}_{m}$ as $\mathcal{E}_{m}(u, v)=-\lambda^{m}{ }^{t} u H_{m} v$. Under Assumption 1),

$$
\inf \left\{\mathcal{E}_{m+1}(u, u),\left.u\right|_{V_{m}}=v\right\}=\mathcal{E}_{m}(v, v), \quad \text { for all } v \in \mathbb{R}^{V_{m}}
$$

so that $\mathcal{E}_{m}\left(\left.u\right|_{V_{m}},\left.u\right|_{V_{m}}\right) \leq \mathcal{E}_{m+1}(u, u)$ for $u \in \mathbb{R}^{V_{m+1}}$. Thus, letting

$\mathcal{F}_{*}=\left\{f \in \mathbb{R}^{V_{*}}: \lim _{m \rightarrow \infty} \mathcal{E}_{m}(f, f)<\infty\right\}, \quad \mathcal{E}(f, g)=\lim _{m \rightarrow \infty} \mathcal{E}_{m}(f, g) \quad \forall f, g \in \mathcal{F}_{*}$,

we have a quadratic form on $V_{*}$. Under Assumption 2), one can define the resistance metric on $V_{*}$ and all the elements in $\mathcal{F}_{*}$ can be extended continuously to $X$. We denote the set of the extended functions as $\mathcal{F}$.

Now, for $\mu_{i}>0$ with $\sum_{i \in S} \mu_{i}=1$, let $\mu$ be a Bernoulli measure on $X$ so that $\mu\left(F_{w_{1} \cdots w_{m}}(X)\right)=\mu_{w_{1}} \cdots \mu_{w_{m}}$. Note that $\mathcal{F} \subset C(X) \subset \mathbb{L}^{2}(X, \mu)$.

Theorem $5.1([35])$ 1 $\quad 1)(\mathcal{E}, \mathcal{F})$ is a local regular Dirichlet form on $\mathbb{L}^{2}(X, \mu)$. It satisfies Lemma 3.1 and the following holds with $\rho_{i}:=\lambda / r_{i}>1$.

$$
\mathcal{E}(f, g)=\sum_{i \in S} \rho_{i} \mathcal{E}\left(f \circ F_{i}, g \circ F_{i}\right) \quad \text { for all } f, g \in \mathcal{F} .
$$


2) Let $\Delta_{\mu}$ be the corresponding self-adjoint operator on $\mathbb{L}^{2}(X, \mu)$. Then $-\Delta_{\mu}$ has the compact resolvent. Define $n^{\mu}(x)=\sharp\left\{\lambda \mid \lambda\right.$ is an eigenvalue of $-\Delta_{\mu} \leq x$. $\}$ and let $d_{s}(\mu)>0$ be the unique number which satisfies $\sum_{i \in S}\left(\mu_{i} / \rho_{i}\right)^{d_{s}(\mu) / 2}=1$. Then,

$$
0<\liminf _{x \rightarrow \infty} n^{\mu}(x) / x^{d_{s}(\mu) / 2} \leq \limsup _{x \rightarrow \infty} n^{\mu}(x) / x^{d_{s}(\mu) / 2}<\infty
$$

3) Let $S>0$ be the unique number which satisfies $\sum_{i \in S} \rho_{i}^{-S}=1$. Then,

$$
\max \left\{d_{s}(\mu): \mu \text { is a Bernoulli measure on } X .\right\}=\frac{2 S}{S+1} \text {. }
$$

Further, maximum is attained by $\mu$ with $\mu_{i}=\rho_{i}^{-S}$ for all $i \in S$.

The former example of $(D, r)$ on the Sierpinski gasket satisfies Assumption $1), 2)$ with $\lambda=5 / 3$. For the case, $\mu$ is $\mu\left(F_{w_{1} \cdots w_{n}}(X)\right)=1 / 3^{n}$ and the middle inequality of (5.2) turns out to be a strict inequality. It is known that for (affine) nested fractals, which is a subclass of p.c.f. self-similar sets with strong symmetry, there exists ( $D, r)$ which satisfies Assumption 1), 2) (cf. [21, 37, 42]). Note that the Sierpinski gasket is an example of an (affine) nested fractal.

We consider $\mu$ with $\mu_{i}=\rho_{i}^{-S}, i \in S$. This case is in the framework of section 3 and $(U V D)$ holds with $V(r)=r^{S}$. Thus, by Theorem 3.1, we have $(U E(h))$ and $(N L E(h))$. In fact, detailed heat kernel estimates are obtained in $[27]$.

Theorem $5.2([27])$. There exists a jointly continuous version of the transition density $p_{t}(x, y)$ such that the following estimate holds.

$$
p_{t}(x, y) \simeq C_{1} t^{-d_{s} / 2} \exp \left(-\left(\frac{R(x, y)^{S+1}}{C_{1} t}\right)^{d_{k(m, t)}^{c}(x, y) /\left(S+1-d_{k(m, t)}^{c}(x, y)\right)}\right)
$$

for all $x, y \in X, 0<t \leq 1$, with the notation of $\simeq$ as in $(3.3)$. Here $d_{k}^{c}(x, y)=$ $\frac{1}{k} \log N_{m+k}(x, y), N_{m}(x, y)$ is the shortest path counting function for the resistance metric at level $m$, and

$$
k=k(m, t):=\inf \left\{j: N_{m+j}(x, y) e^{-(S+1)(m+j)}<t\right\} .
$$

See [27] for details. Note that in general we cannot obtain uniform estimates for $d_{k}^{c}(x, y)$ as shown in [27] section 6 . Thus, we cannot get $(L E(h))$ in general as mentioned in section 3 . 
For (affine) nested fractals, it is known that there exists a geodesic $d$ such that $R(x, y) \simeq d(x, y)^{\theta}$ holds for some $\theta \leq 1$ and

$$
p_{t}(x, y) \simeq C_{1} t^{-S /(S+1)} \exp \left(-\left(\frac{R(x, y)^{S+1}}{C_{1} t}\right)^{d_{c}^{r} /\left(S+1-d_{c}^{r}\right)}\right),
$$

for all $x, y \in X, 0<t \leq 1$ where $d_{c}^{r}=1 / \theta$ (see [21,37]). When $\theta<1$, this estimate is different from $(U E(h))+(L E(h))$ with $V(r)=r^{S}$. This means, $(L E(h))$ and $(C C)$ do not hold and $(U E(h))$ is not the best estimate when $\theta<1$. For the case of Brownian motion on the Sierpinski gasket, which is constructed from the former example of $(D, r), \theta=\log (5 / 3) / \log 2<1$.

By these results, we see that geodesic is needed in order to obtain two-sided heat kernel estimates, whereas for parabolic Harnack inequalities resistance metric seems to be more useful when it is properly defined.

We note that the 2-dimensional Sierpinski carpet (which is not a p.c.f. self-similar set, cf. $[7,40])$ is also an example that we can apply our results.

\section{Acknowledgement}

The author thanks Martin Barlow and Thierry Coulhon for letting him refer and use the results in [12] before the preprint is ready. He is grateful to Jun Kigami and Laurent Saloff-Coste for stimulating discussions.

\section{References}

[1] Aronson, D. G., Bounds on the fundamental solution of a parabolic equation, Bull. Amer. Math. Soc., 73 (1967), 890-896.

[2] Barlow, M. T., Diffusions on fractals, in Lectures in Probability Theory and Statistics: Ecole d'été de probabilités de Saint-Flour XXV, 1-121, Lect. Notes Math., 1690, Springer, New York, 1998.

[3] Which values of the volume growth and escape time exponent are possible for a graph? Rev. Math. Iberoamericana, 20 (2004), 1-31.

[4] - Heat kernels and sets with fractal structure, 11-40, Contemp. Math., 338, A.M.S., Providence, RI, 2003.

[5] - Some remarks on the elliptic Harnack inequality, Bull. London Math. Soc., to appear.

[6] _ Anomalous diffusion and stability of Harnack inequalities, Surv. Differ. Geom. IX, to appear.

[7] Barlow, M. T. and Bass, R. F., Brownian motion and harmonic analysis on Sierpinski carpets, Canad. J. Math., 51 (1999), 673-744.

[8] _ Stability of parabolic Harnack inequalities, Trans. Amer. Math. Soc., 356 (2003), 1501-1533.

[9] Random walks on graphical Sierpinski carpets, In: Random walks and discrete potential theory (Cortona, 1997), 26-55, Sympos. Math., XXXIX, Cambridge Univ. Press, Cambridge, 1999. 
[10] Barlow, M. T., Bass, R. F. and Kumagai, T., Stability of parabolic Harnack inequalities on measure metric spaces, in preparation.

[11] Barlow, M. T., Coulhon, T. and Grigor'yan, A., Manifolds and graphs with slow heat kernel decay, Invent. Math., 144 (2001), 609-649.

[12] Barlow, M. T., Coulhon, T. and Kumagai, T., Characterization of sub-Gaussian heat kernel estimates on strongly recurrent graphs, Preprint.

[13] Bass, R. F., Diffusions and elliptic operators, Springer, New York, 1997.

[14] Biroli, M. and Mosco, U. A., Saint-Venant type principle for Dirichlet forms on discontinuous media, Ann. Mat. Pura Appl., 169 (1995), 125-181.

[15] Coulhon, T., Off-diagonal heat kernel lower bounds without Poincaré, J. London Math. Soc, 68 (2003), 795-816.

[16] Coulhon, T. and Saloff-Coste, L., Minorations pour les chaines de Markov unidimensionnelles, Probab. Theory Related Fields, 97 (1993), 423-431.

[17] Variétés riemanniennes isométriques á l'infini, Rev. Mat. Iberoamericana, 11 (1995), 687-726.

[18] Davies, E. B., Heat kernels and spectral theory, Cambridge University Press, Cambridge, 1989.

[19] Delmotte, T., Parabolic Harnack inequality and estimates of Markov chains on graphs, Rev. Math. Iberoamericana, 15 (1999), 181-232.

[20] Fabes, E. B. and Stroock, D. W., A new proof of Moser's parabolic Harnack inequality using the old ideas of Nash, Arch. Rational Mech. Anal., 96 (1986), 327-338.

[21] Fitzsimmons, P. J., Hambly, B. M. and Kumagai, T., Transition density estimates for Brownian motion on affine nested fractals, Commun. Math. Phys., 165 (1994), 595-620.

[22] Fukushima, M., Oshima, Y. and Takeda, M., Dirichlet Forms and Symmetric Markov Processes, de Gruyter, Berlin, 1994.

[23] Grigor'yan, A., The heat equation on non-compact Riemannian manifolds, (in Russian) Matem. Sbornik., 182 (1991), 55-87. (English transl.) Math. USSR Sb., 72 (1992), 47-77.

[24] Grigor'yan, A. and Telcs, A., Sub-Gaussian estimates of heat kernels on infinite graphs, Duke Math. J., 109 (2001), 451-510.

[25] Harnack inequalities and sub-Gaussian estimates for random walks, Math. Annal., 324 (2002), 521-556.

[26] $\quad$ in preparation.

[27] Hambly, B. M. and Kumagai, T., Transition density estimates for diffusions on post critically finite fractals, Proc. London Math. Soc., 78 (1999), 431-458.

[28] _ Heat kernel estimates for symmetric random walks on a class of fractal graphs and stability under rough isometries, Proc. Sympos. Pure Math., to appear.

[29] Havlin, S. and Ben-Avraham, D., Diffusion in disordered media, Adv. Phys., 36 (1987), 695-798.

[30] Hebisch, W. and Saloff-Coste, L., On the relation between elliptic and parabolic Harnack inequalities, Ann. Inst. Fourier (Grenoble), 51 (2001), 1437-1481.

[31] Jones, O. D., Transition probabilities for the simple random walk on the Sierpinski graph, Stochastic Process. Appl., 61 (1996), 45-69.

[32] Kanai, M., Rough isometries, and combinatorial approximations of geometries of noncompact riemannian manifolds, J. Math. Soc. Japan, 37 (1985), 391-413.

[33] Analytic inequalities, and rough isometries between non-compact riemannian manifolds, 122-137, Lect. Notes Math., 1201, Springer, Berlin, 1986.

[34] Kigami, J., Harmonic calculus on limits of networks and its application to dendrites, $J$. Funct. Anal., 128 (1995), 48-86.

[35] _ Analysis on Fractals, Cambridge Univ. Press, Cambridge, 2001.

[36] - Harmonic analysis for resistance forms, J. Funct. Anal., 204 (2003), 399-444.

[37] Kumagai, T., Estimates of transition densities for Brownian motion on nested fractals, Probab. Theory Relat. Fields, 96 (1993), 205-224.

[38] Stochastic processes on fractals and related topics, (in Japanese) Sugaku, 49 (1997), 158-172, (English transl.) Sugaku Expositions, 13 (2000), 55-71. 
[39] Kusuoka, S. and Stroock, D. W., Applications of the Malliavin calculus. III, J. Fac. Sci. Univ. Tokyo Sect. IA Math., 34 (1987), 391-442.

[40] Kusuoka, S. and Zhou, X. Y., Dirichlet form on fractals: Poincaré constant and resistance, Probab. Theory Relat. Fields, 93 (1992), 169-196.

[41] Li, P. and Yau, S. T., On the parabolic kernel of the Schrödinger operator, Acta Math., 156 (1986), 153-201.

[42] Lindstrøm, T., Brownian motion on nested fractals, Memoirs Amer. Math. Soc., 83 (1990), 420.

[43] Moser, J., On Harnack's inequality for elliptic differential equations, Comm. Pure Appl. Math., 14 (1961), 577-591.

[44] On Harnack's inequality for parabolic differential equations, Comm. Pure Appl. Math., 17 (1964), 101-134.

[45] Nash, J., Continuity of solutions of parabolic and elliptic equations, Amer. Math. J., 80 (1958), 931-954.

[46] Saloff-Coste, L., A note on Poincaré, Sobolev and Harnack inequality, Internat. Math. Res. Notices, 2 (1992), 27-38.

[47] _ Aspects of Sobolev-type inequalities, Cambridge Univ. Press, Cambridge, 2002.

[48] Analysis on Riemannian co-compact covers, Surv. Differ. Geom. IX, to appear.

[49] Strichartz, R. S., Analysis on fractals, Notices Amer. Math. Soc., 46 (1999), 1199-1208.

[50] Sturm, K. T., Analysis on local Dirichlet spaces-II. Gaussian upper bounds for the fundamental solutions of parabolic Harnack equations, Osaka J. Math., 32 (1995), 275312 .

[51] - Analysis on local Dirichlet spaces-III. the parabolic Harnack inequality, J. Math. Pure Appl., 75 (1996), 273-297.

[52] Telcs, A., Volume and time doubling of graphs and random walks: the strongly recurrent case, Comm. Pure Appl. Math., 54 (2001), 975-1018.

[53] Local sub-Gaussian estimates on graphs: the strongly recurrent case, Elect. J. Probab., 6 (2001), 1-33. 